\title{
Asymptotic properties of Born-improved amplitudes with gauge bosons in the final state
}

\author{
Joannis Papavassiliou \\ Theory Division, CERN, CH-1211 Geneva 23, Switzerland \\ (Received 8 February 1999; published 2 August 1999)
}

\begin{abstract}
For processes with gauge bosons in the final state we show how to continuously connect with a single Born-improved amplitude the resonant region, where resummation effects are important, with the asymptotic region far away from the resonance, where the amplitude must reduce to its tree-level form. While doing so all known field-theoretical constraints are respected, most notably gauge invariance, unitarity and the equivalence theorem. The calculations presented are based on the process $f \bar{f} \rightarrow Z Z$, mediated by a possibly resonant Higgs boson; this process captures all the essential features, and can serve as a prototype for a variety of similar calculations. By virtue of massive cancellations the resulting closed expressions for the differential and total cross sections are particularly compact. [S0556-2821(99)08213-2]

PACS number(s): 11.10.Jj
\end{abstract}

\section{INTRODUCTION}

The physics of unstable particles in general [1] and the computation of resonant transition amplitudes in particular [2] has attracted significant attention in recent years, because it is both phenomenologically relevant and theoretically challenging. The main theoretical difficulty arises from the fact that in the context of non-Abelian gauge theories the standard Breit-Wigner resummation used for regulating physical amplitudes near resonances is at odds with gauge invariance, unitarity, and the equivalence theorem [3]. Consequently, the resulting Born-improved amplitudes in general fail to capture faithfully the underlying dynamics.

A solution to this problem has been accomplished at the one-loop level $[4,5]$ by resorting to the reorganization of perturbation theory implemented by the pinch technique (PT) $[6,7]$. The resummation formalism based on the latter method satisfies a set of crucial physical requirements, and provides a self-consistent framework for dealing with resonant transition amplitudes. The main thrust of this diagrammatic method is to exploit the properties built into physical amplitudes in order to construct off-shell Green's functions with the following properties: (i) they are independent of the gauge-fixing parameter; (ii) they satisfy naive (ghost-free) tree-level Ward identities (WI's) instead of the usual Slavnov-Taylor identities; (iii) they display physical thresholds only [4]; (iv) they satisfy individually the optical and equivalence theorems $[4,8,5]$; (v) they are analytic functions of the kinematic variables; (vi) the effective two-point functions constructed are universal (process-independent) [9], Dyson-resummable $[4,10]$, and do not shift the position of the gauge-independent complex pole $[4,11]$.

From the phenomenological point of view the upshot of the above framework is to construct Born-improved amplitudes in which all relevant physical information has been encoded. This in turn is useful for the detailed study of the physical properties of particles, most importantly the correct extraction of their masses, widths, and line shapes. The precise measurement of the mass and the width of the $W$ gauge boson for example is of fundamental physical importance. At present $W$ bosons are produced at the Fermilab Tevatron (single $W$ production) and at the CERN $e^{+} e^{-}$collider LEP2
( $W$ pair-production), whereas large numbers of $W$ bosons are expected to be produced at the CERN Large Hadron Collider (LHC). In addition, muon colliders are scheduled to operate as Higgs factories for intermediate energies of about 500 $\mathrm{GeV}$, and copious amounts of Higgs bosons through resonant s-channel production are expected [12].

Given the importance of Born-improved amplitudes, one must study their properties further. One open question in this context is how to connect smoothly resonant with asymptotic regions. On physical grounds one expects that far from the resonance the Born-improved amplitude must behave exactly as its tree-level counterpart; in fact, a self-consistent resummation formalism should have this property built in, i.e. far from resonance one should recover the correct high energy behavior without having to re-expand the Born-improved amplitude perturbatively. Recovering the correct asymptotic behavior is particularly tricky however when the final particles are gauge bosons. In order to accomplish this, in addition to the correct one-loop (running) width, the appropriate one-loop vertex corrections must be supplemented; these vertex corrections and the width must be related by a crucial tree-level Ward identity. In practice this WI ensures that massive cancellations which take place at tree-level will still go through after the Born-amplitude has been "dressed."

The need for preserving tree-level Ward identities when dealing with gauge bosons in the final state has been emphasized from various points of view in the recent literature. As was pointed out first in [13] in the context of the process $q \bar{q}^{\prime} \rightarrow l \nu \gamma$, maintaining the electromagnetic gauge invariance associated with the outgoing photon necessitates such a WI relating the running (fermionic) width coming from the $W$ self-energy and the $W W \gamma$ vertex containing a fermionic triangle. The phenomenological implications of this observation were further studied in [14], where the complete set of fermionic corrections was taken into account. ${ }^{1}$ The nonAbelian case has been addressed in [4] using the PT; the

\footnotetext{
${ }^{1}$ In the fermionic case both the gauge-fixing parameter independence of the result and the preservation of the WI are automatic, because these corrections are Abelian-like.
} 
non-trivial point in this context is to construct one-loop running widths and one-loop three-boson vertices which are independent of the gauge-fixing parameter, and at the same time satisfy tree-level WI. ${ }^{2}$ Finally, as was shown in [5] these WI are crucial for satisfying the equivalence theorem before and after resummation. However, to date it has not been demonstrated explicitly (i) how the need for maintaining the tree-level WI manifests itself at the level of the crosssection, for both Abelian (fermionic) and non-Abelian (bosonic) corrections, (ii) what is the precise field-theoretical mechanism which restores the correct high-energy behavior; as we will see in detail, the WI by itself is neither a necessary nor a sufficient condition for recovering the correct asymptotic behavior, and must be combined with additional requirements, (iii) whether the PT algorithm has all aforementioned necessary requirements built in it.

In this paper we will address the issues listed above. In particular, we will show with detailed analytic calculations that the Born-improved amplitudes constructed by means of the PT resummation algorithm not only encodes correctly the effects on and around the resonant but also far from it. To study the above points we will calculate the (resonant) Higgs boson contributions to the process $f \bar{f} \rightarrow Z Z$. The motivation for turning to this particular process is three-fold. First, from the theoretical point of view this process contains all necessary features, without additional technical complications; for example, unlike the $f \bar{f} \rightarrow W^{+} W^{-}$it does not contain any non-resonant (non-Higgs boson related) background due to an $s$-channel $\gamma$ and $Z$. Second, the Higgs boson self-energy and vertex receives contributions from loops containing fermions, scalars, and gauge bosons. Therefore this process can serve as a prototype for studying the relevant issues. Third, the resonant process $f \bar{f} \rightarrow Z Z$ may be intrinsically interesting for muon colliders, if the standard model Higgs boson turns out to be heavier than $2 M_{Z}$. Therefore, the exact closed expressions for the Born-improved amplitude presented here may be useful for studying various properties of this process in detail.

The paper is organized as follows: In Sec. II we derive closed expressions for the differential and total cross sections away from the resonance, and establish their high energy behavior. In Sec. III we calculate the same cross sections where we now account for resonant effects; in particular we derive closed expressions for an arbitrary running width and an arbitrary form of the $H Z Z$ vertex compatible with Lorentz invariance and $C P$ symmetry. We then analyze in detail the mechanism which enforces the correct high energy behavior of the Born-improved amplitude when the PT width and vertex are used. In Sec. IV we show how the mechanism of the previous section can be realized explicitly in the PT context. In Sec. V we present our conclusions. Finally, various useful formulas are presented in an Appendix. Throughout this work we only consider the absorptive (imaginary) contributions to the width and the vertex; they constitute the mini-

\footnotetext{
${ }^{2}$ In the context of the background field method [15] or the axial gauges [16] only the latter property is guaranteed.
}

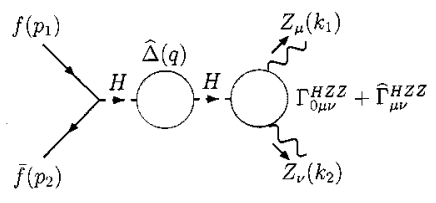

(a)

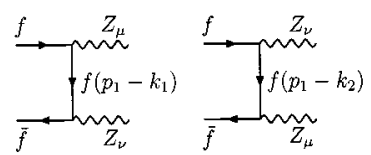

(b)

(c)
FIG. 1. The Born-improved amplitude for the process $f \bar{f} \rightarrow Z Z$.

mum amount of "dressing" necessary in order to regulate the resonant amplitude. The real (dispersive) corrections can also be included in a systematic way, but this task is beyond the scope of the present work.

\section{THE DIFFERENTIAL AND TOTAL CROSS SECTIONS}

In this section we present closed expressions for the treelevel differential and total cross sections for the process $f\left(p_{1}\right) \bar{f}\left(p_{2}\right) \rightarrow Z\left(k_{1}\right) Z\left(k_{2}\right)$ and study their behavior in the limit where the center-of-mass energy is much larger than any other mass scale. The purpose is twofold: (i) we show that if the cancellations of the PT are implemented before the calculation the resulting expressions are rather compact; (ii) based on these closed expressions we can easily establish the behavior of the cross section far from resonance.

The tree-level transition amplitude $\mathcal{T}_{\mu \nu}$ for the process $f\left(p_{1}\right) \bar{f}\left(p_{2}\right) \rightarrow Z\left(k_{1}\right) Z\left(k_{2}\right)$ is the sum of an $s$-, a $t$-, and a $u$ channel contribution (Fig. 1), denoted by $\mathcal{T}_{s \mu \nu}, \mathcal{T}_{t \mu \nu}$, and $\mathcal{T}_{u \mu \nu}$, respectively, given by

$$
\begin{aligned}
& \mathcal{T}_{s \mu \nu}=\overline{\mathrm{v}}\left(p_{2}\right) \Gamma_{0}^{H f \bar{f}} u\left(p_{1}\right) \Delta_{0}(s) \Gamma_{0 \mu \nu}^{H Z Z}, \\
& \mathcal{T}_{t \mu \nu}=\overline{\mathrm{v}}\left(p_{2}\right) \Gamma_{0 \nu}^{Z f \bar{f}} \frac{1}{p_{1}-k_{1}-m_{f}} \Gamma_{0 \mu}^{Z f \bar{f}} u\left(p_{1}\right), \\
& \mathcal{T}_{u \mu \nu}=\overline{\mathrm{v}}\left(p_{2}\right) \Gamma_{0 \mu}^{Z f \bar{f}} \frac{1}{p_{1}-k_{2}-m_{f}} \Gamma_{0 \nu}^{Z f \bar{f}} u\left(p_{1}\right) .
\end{aligned}
$$

Here, $s=\left(p_{1}+p_{2}\right)^{2}=\left(k_{1}+k_{2}\right)^{2}$ is the center-of-mass energy squared, $\quad \Gamma_{0 \mu \nu}^{H Z Z}=\left(i g_{w} M_{Z} / c_{w}\right) g_{\mu \nu}, \quad \Gamma_{0}^{H f \bar{f}}$ $=-i g_{w} m_{f} /\left(2 M_{W}\right)$ and $\Gamma_{0 \mu}^{Z f \bar{f}}=-i g_{w} /\left(2 c_{w}\right) \gamma_{\mu}\left[T_{z}^{f}\left(1-\gamma_{5}\right)\right.$ $\left.-2 Q_{f} s_{w}^{2}\right]$, with $c_{w}=\sqrt{1-s_{w}^{2}}=M_{W} / M_{Z}$, are the tree-level $H Z Z, H f \bar{f}$ and $Z f \bar{f}$ couplings, respectively, $Q_{f}$ is the electric charge of the fermion $f$, and $T_{z}^{f}$ its $z$-component of the weak isospin. Away from the resonance the propagator for the Higgs boson is given by the usual tree-level expression $\Delta_{0}(s)=\left(s-M_{H}^{2}\right)^{-1}$. For on shell $Z$ bosons, i.e., $k_{1}^{2}=k_{2}^{2}$ $=M_{Z}^{2}$, the vertex $\Gamma_{0 \mu \nu}^{H Z Z}$ satisfies the following WI:

$$
k_{1}^{\mu} k_{2}^{\nu} \Gamma_{0 \mu \nu}^{H Z Z}=\frac{i g_{w} M_{Z}}{2 c_{w}}\left[\Delta_{0}^{-1}(s)+\left(M_{H}^{2}-2 M_{Z}^{2}\right)\right],
$$

which, as we will see, controls the high energy behavior of the tree-level amplitude.

The Mandelstam variables $t$ and $u$ are given by 


$$
\begin{aligned}
& t=\left(k_{1}-p_{1}\right)^{2}=\left(p_{2}-k_{2}\right)^{2}=-\frac{1}{4}\left(\beta_{Z}^{2}+\beta_{f}^{2}-2 z x\right) s, \\
& u=\left(k_{1}-p_{2}\right)^{2}=\left(p_{1}-k_{2}\right)^{2}=-\frac{1}{4}\left(\beta_{Z}^{2}+\beta_{f}^{2}+2 z x\right) s,
\end{aligned}
$$

where $x \equiv \cos \theta$ is the center-of-mass scattering angle, and

$$
\beta_{Z}=\sqrt{1-\frac{4 M_{Z}^{2}}{s}}, \quad \beta_{f}=\sqrt{1-\frac{4 m_{f}^{2}}{s}}, \quad z=\beta_{Z} \beta_{f} .
$$

The squared matrix element $|\overline{\mathcal{M}}|^{2}$ averaged over initial and final polarization states is given by

$$
\begin{aligned}
\overline{|\mathcal{M}|^{2}}= & \frac{1}{4} \sum_{s_{1}, s_{2}}\left[\bar{v} T_{\mu \nu} u\right]\left(g^{\mu \mu^{\prime}}-\frac{k_{1}^{\mu} k_{1}^{\mu^{\prime}}}{M_{Z}^{2}}\right)\left(g^{\nu \nu^{\prime}}-\frac{k_{2}^{\nu} k_{2}^{\nu^{\prime}}}{M_{Z}^{2}}\right) \\
& \times\left[\bar{u} T_{\mu^{\prime} \nu^{\prime}}^{\dagger} v\right],
\end{aligned}
$$

and the unpolarized differential cross section for leptons in the initial state ${ }^{3}$ reads

$$
\frac{d \sigma}{d x}=\frac{1}{32 \pi} \frac{\beta_{Z}}{\beta_{f}} \frac{1}{s} \overline{|\mathcal{M}|^{2}}
$$

For the actual calculation it is convenient to write $\overline{|\mathcal{M}|^{2}}$ as the sum of six sub-amplitudes distinguished by their dependence on the three Mandelstam variables $s, t$, and $u$. In carrying out this decomposition we follow the method explained in detail in [8]; in particular, we carry out analytically a large number of cancellations between terms originating from the longitudinal pieces $k_{1}^{\mu} k_{1}^{\mu^{\prime}} / M_{Z}^{2}$ and $k_{2}^{\nu} k_{2}^{\nu^{\prime}} / M_{Z}^{2}$ appearing on the left-hand side of Eq. (2.5). These cancellations are carried out systematically by resorting to the PT reorganization of the amplitude, i.e. we use the treelevel Ward identity obeyed by the amplitude in order to extract $s$-channel-like pieces from $t$ and $u$ graphs, which cancel against analogous contributions coming from the usual $s$-channel graph. Specifically, we start from the following elementary WI satisfied by the two sub-amplitudes:

$$
\begin{gathered}
k_{1}^{\mu} k_{2}^{\nu} \mathcal{T}_{s \mu \nu}=\mathcal{T}_{s}+\mathcal{T}_{P}, \\
k_{1}^{\mu} k_{2}^{\nu}\left(\mathcal{T}_{t \mu \nu}+\mathcal{T}_{u \mu \nu}\right)=M_{Z}^{2}\left(\mathcal{T}_{t}+\mathcal{T}_{u}\right)-\mathcal{T}_{P},
\end{gathered}
$$

with

$$
\mathcal{T}_{P}=\overline{\mathrm{v}}\left(p_{2}\right) \Gamma_{0}^{H f \bar{f}} u\left(p_{1}\right) \Delta_{0}(s)\left(\frac{i g_{w} M_{Z}}{2 c_{w}}\right) \Delta_{0}^{-1}(s)
$$

and

${ }^{3}$ If the initial fermions are quarks we must multiply by a factor of $\frac{1}{3}$.

$$
\begin{aligned}
& \mathcal{T}_{s}=\overline{\mathrm{v}}\left(p_{2}\right) \Gamma_{0}^{H f \bar{f}} u\left(p_{1}\right) \Delta_{0}(s)\left(\frac{i g_{w} M_{Z}}{2 c_{w}}\right)\left(M_{H}^{2}-2 M_{Z}^{2}\right), \\
& \mathcal{T}_{t}=\overline{\mathrm{v}}\left(p_{2}\right) \Gamma_{0}^{G^{0} f \bar{f}} \frac{1}{p_{1}-k_{1}-m_{f}} \Gamma_{0}^{G^{0} f \bar{f}} u\left(p_{1}\right), \\
& \mathcal{T}_{u}=\overline{\mathrm{v}}\left(p_{2}\right) \Gamma_{0}^{G^{0} f \bar{f}} \frac{1}{p_{1}-k_{2}-m_{f}} \Gamma_{0}^{G^{0} f \bar{f}^{\prime}} u\left(p_{1}\right)
\end{aligned}
$$

where $\Gamma^{G^{0} f \bar{f}}=-g_{w}\left(m_{f} / M_{W}\right) T_{z}^{f} \gamma_{5}$ is the coupling of the neutral Goldstone boson $G^{0}$ to the fermions. Then, by adding both parts of Eq. (2.7) we see that the $\mathcal{T}_{P}$ terms cancel on the right-hand side (RHS) and we are left exactly with what one expects from the (generalized) equivalence theorem [5].

After carrying out the above cancellations, a straightforward calculation shows that the differential cross section reads

$$
\begin{aligned}
\frac{d \sigma}{d x}= & \left(\frac{\pi}{64}\right)\left(\frac{\alpha_{w}^{2}}{c_{w}^{4}}\right)\left(\frac{\beta_{Z}}{\beta_{f}}\right)\left(\frac{1}{s}\right)\left[M_{s s}+M_{s t}\right. \\
& \left.+M_{s u}+M_{t t}+M_{u u}+M_{u t}\right],
\end{aligned}
$$

where $\alpha_{w}=g_{w}^{2} /(4 \pi)$, and

$$
\begin{aligned}
& M_{s s}=s \Delta_{0}^{2} m_{f}^{2} \beta_{f}^{2} f_{1}, \\
& M_{s t}=\frac{2 s}{\left(t-m_{f}^{2}\right)} \Delta_{0} m_{f}^{2}\left[f_{2} \beta_{f}^{2}-f_{3} z x\right], \\
& M_{s u}=\frac{2 s}{\left(u-m_{f}^{2}\right)} \Delta_{0} m_{f}^{2}\left[f_{2} \beta_{f}^{2}+f_{3} z x\right], \\
& M_{t t}=\frac{1}{2} \frac{s^{2}}{\left(t-m_{f}^{2}\right)^{2}}\left[f_{4} z^{2} x^{2}-f_{5} z x+f_{6}\right], \\
& M_{u u}=\frac{1}{2} \frac{s^{2}}{\left(u-m_{f}^{2}\right)^{2}}\left[f_{4} z^{2} x^{2}+f_{5} z x+f_{6}\right], \\
& M_{u t}=\frac{1}{2} \frac{s^{2}}{\left(u-m_{f}^{2}\right)\left(t-m_{f}^{2}\right)}\left[f_{7} z^{2} x^{2}+f_{8}\right],
\end{aligned}
$$

with

$$
\begin{aligned}
& f_{1}=12-8 \frac{s}{M_{Z}^{2}}+4 \frac{M_{H}^{2}}{M_{Z}^{2}}+\frac{M_{H}^{4}}{M_{Z}^{4}}, \\
& f_{2}=3-4 a_{f}-\frac{s}{M_{Z}^{2}}, \\
& f_{3}=1+4 a_{f}-\frac{s}{M_{Z}^{2}}+\frac{m_{f}^{2}}{M_{Z}^{4}}\left(M_{H}^{2}+2 M_{Z}^{2}\right),
\end{aligned}
$$




$$
\begin{aligned}
f_{4}= & -\left(2 a_{f}^{2}+3 a_{f}+\frac{1}{8}\right)-\frac{1}{2} \frac{m_{f}^{2}}{M_{Z}^{2}}\left(4 a_{f}+1\right)-\frac{1}{2} \frac{m_{f}^{4}}{M_{Z}^{4}}, \\
f_{5}= & \frac{m_{f}^{2}}{s}\left(8 a_{f}^{2}-4 a_{f}+\frac{9}{2}\right), \\
f_{6}= & \left(2 a_{f}^{2}+3 a_{f}+\frac{1}{8}\right) \beta_{Z}^{2}+\frac{m_{f}^{2}}{s}\left(8 a_{f}^{2}-28 a_{f}+\frac{5}{2}\right) \\
& +\frac{1}{2} \frac{m_{f}^{2}}{M_{Z}^{2}}\left(4 a_{f}+1\right)-\frac{m_{f}^{2} M_{Z}^{2}}{s^{2}}\left(48 a_{f}^{2}-56 a_{f}+3\right) \\
& -\frac{2 m_{f}^{4}}{s^{2}}\left(16 a_{f}^{2}-16 a_{f}+3\right)+\frac{1}{2} \frac{m_{f}^{4}}{M_{Z}^{4}}-\frac{2 m_{f}^{4}}{M_{Z}^{2} s}, \\
& -\frac{m_{f}^{2}}{M_{Z}^{2}}\left(1-4 a_{f}\right)+\frac{m_{f}^{4}}{M_{Z}^{4}}, \\
& +\frac{m_{f}^{2}}{M_{Z}^{2}}\left(4 a_{f}-1\right)-\frac{4 m_{f}^{4}}{s^{2}}\left(16 a_{f}^{2}-16 a_{f}-1\right) \\
f_{8}= & \frac{m_{f}^{2}}{s}\left(16 a_{f}^{2}-56 a_{f}-3\right)+\frac{16 M_{Z}^{2}}{s}\left(2 a_{f}^{2}+3 a_{f}+\frac{1}{8}\right) \\
& \\
f_{7}^{2} &
\end{aligned}
$$

where we have defined $a_{f}=\left(T_{z}^{f}-2 Q_{f} s_{w}^{2}\right)^{2}$.

The total cross section is given by

$$
\sigma=\frac{1}{2 !} \int_{-1}^{1} d x\left(\frac{d \sigma}{d x}\right)
$$

where the statistical factor $1 / 2$ ! is due to the two identical $Z$ bosons in the final state. After carrying out the angular integration $\sigma$ is given by

$\sigma=\left(\frac{\pi}{128}\right)\left(\frac{\alpha_{w}^{2}}{c_{w}^{4}}\right)\left(\frac{\beta_{Z}}{\beta_{f}}\right)\left(\frac{1}{s}\right)\left[\sigma_{s s}+\sigma_{s t}+\sigma_{s u}+\sigma_{t t}+\sigma_{u u}+\sigma_{u t}\right]$,

where

$$
\begin{gathered}
\sigma_{s s}=2 s \Delta_{0}^{2} m_{f}^{2} \beta_{f}^{2} f_{1}, \\
\sigma_{s t}=\sigma_{s u}=-8 \Delta_{0} m_{f}^{2}\left[f_{3}-\frac{V}{2 z}\left(\beta_{f}^{2} f_{2}+y f_{3}\right)\right], \\
\sigma_{t t}=\sigma_{u u}=\frac{4}{y^{2}-z^{2}}\left[z^{2} f_{4}+y f_{5}+f_{6}\right] \\
+8 f_{4}-\frac{2}{z}\left(f_{5}+2 y f_{4}\right) V
\end{gathered}
$$

$$
\sigma_{u t}=\frac{2}{z y}\left[z(z-2 y) f_{7}+\left(y^{2} f_{7}+f_{8}\right) V\right],
$$

and

$$
y=-\frac{1}{2}\left(1+\beta_{Z}^{2}\right), \quad V=\ln \left|\frac{y+z}{y-z}\right|,
$$

and we have used that $t-m_{f}^{2}=(s / 2)(y+z x)$ and $u-m_{f}^{2}$ $=(s / 2)(y-z x)$.

The following comments are now in order:

(i) By virtue of the extensive cancellations described at the beginning of this section, the resulting expressions for the differential and total cross sections are particularly compact.

(ii) For $m_{f}=0$ the differential and total cross sections given above reduce to the expressions given in Eq. (3.5) and Eq. (3.7) of [17], respectively.

(iii) Notice that all sub-amplitudes $f_{i}$ given in Eq. (2.12) behave at most as constants for large $s$. This is a generic feature of the PT reorganization of the amplitude, as was demonstrated first in [8] for the case of $e^{+} e^{-} \rightarrow W^{+} W^{-}$.

(iv) It is straightforward to verify that in the limit $s \gg \mu^{2}$, where $\mu$ is any of the particle masses in the process, i.e. $m_{f}^{2}$, $M_{z}^{2}, M_{W}^{2}$, and $M_{H}^{2}$, we have that $f_{2}=f_{3}=\frac{1}{8} f_{1}, f_{5}=0, f_{4}$ $=-f_{6}$ and $f_{7}=-f_{8}$. In that limit we obtain

$$
\begin{aligned}
& \sigma_{s s}+\sigma_{s t}+\sigma_{s u}=\mathcal{O}\left(\frac{\mu^{2}}{s}\right), \\
& \sigma_{t t}+\sigma_{u u}+\sigma_{u t}=8\left|f_{4}\right| \ln \left(s / m_{f}^{2}\right)+\cdots,
\end{aligned}
$$

where the ellipsis denote terms which are at most constants. Consequently, for large $s$ the total cross section is the (manifestly positive) quantity

$$
\sigma=\left(\frac{\pi}{16}\right)\left(\frac{\alpha_{w}^{2}}{c_{w}^{4}}\right)\left(\frac{1}{s}\right)\left|f_{4}\right| \ln \left(s / m_{f}^{2}\right)
$$

We see that, as is expected on physical grounds, the asymptotic behavior of the cross section is determined by the "genuine" $t$-and $u$-channel terms, i.e. the $t$-and $u$-channel terms remaining after the cancellations of the longitudinal polarization momenta has been carried out.

(v) The expression for $\sigma_{s s}$ is identical to the imaginary (absorptive) part of the gauge-invariant set of one-loop selfenergy-like graphs involving two virtual $Z$ bosons (together with the corresponding Goldstone bosons and ghosts) derived in [5], given also in Sec. IV of the present paper.

\section{THE BORN-IMPROVED AMPLITUDE}

In this section we will recompute the amplitude for the process $f\left(p_{1}\right) \bar{f}\left(p_{2}\right) \rightarrow Z\left(k_{1}\right) Z\left(k_{2}\right)$ using a generic parametrization for the width of the Higgs boson, and for the $H Z Z$ vertex. This calculation will show quantitatively how the high energy behavior of the amplitude is altered if the parametrization of the width and the vertex is kept arbitrary, 
and the precise role of the WI will be analyzed. In addition it will be shown that if the PT width and vertex are used, the correct high energy behavior will emerge.

In the vicinity of the Higgs boson resonance, i.e. for $s$ $\sim M_{H}^{2}$, the amplitude $\mathcal{T}_{s \mu \nu}$ given in Eq. (2.1) diverges, and must be regulated by introducing a width in the Higgs boson propagator. In particular we must replace the tree-level $\Delta_{0}$ by a $\Delta$ of the form

$$
\Delta=\left[s-M_{H}^{2}+i \Im m \Pi(s)\right]^{-1},
$$

where $\Pi(s)$ is the (appropriately defined) one-loop selfenergy of the Higgs boson. For the purposes of this work it is convenient to introduce the dimensionless quantity $L(s)$ as follows:

$$
\Im m \Pi(s)=s L(s) .
$$

The correct one-loop expressions for the various decay channels contributing to $\Im m \Pi(s)$ have been derived in [5] and are also reported in the next section. However for the purposes of this calculation $L$ will be treated as an arbitrary parameter. Similarly, the most general tensorial decomposition of the HZZ vertex, where the two on-shell $Z$ are assumed to be contracted with their corresponding polarization vectors, reads

$$
\begin{aligned}
G_{\mu \nu}^{H Z Z}(q, p, k) & =\frac{i g_{w} M_{Z}}{c_{w}}\left[(1+A(s)) g_{\mu \nu}+B(s) \frac{q_{\mu} q_{\nu}}{q^{2}}\right] \\
& =\Gamma_{0 \mu \nu}^{H Z Z}+\hat{\Gamma}_{\mu \nu}^{H Z Z} .
\end{aligned}
$$

Notice that in general the $C P$-violating form-factor proportional to $\epsilon_{\mu \nu \rho \sigma} k_{1}^{\rho} k_{2}^{\sigma}$ may appear in Eq. (3.3), but it vanishes at one-loop in the standard model. ${ }^{4}$ The explicit one-loop expressions (see Fig. 2) for $A$ and $B$ are also computable by means of the PT, and will be presented in the next section, but for the purposes of this section they too will be treated as arbitrary quantities.

Next we calculate the differential and total cross sections using the above modified propagator and vertex. The new differential cross section is obtained from Eq. (2.11) after replacing $M_{s s}, M_{s t}$ and $M_{s u}$ by the the modified amplitudes $\hat{M}_{s s}, \hat{M}_{s t}$, and $\hat{M}_{s u}$, respectively, given by

\footnotetext{
${ }^{4}$ There are also contributions originating from the one-loop mixing between the Higgs boson and the $Z$ boson. Such contributions have been treated correctly within the PT framework in $[18,19]$. It is easy to verify from the expressions given in Eq. (6) of the first reference in [19] that all such mixing contributions are non-resonant in the entire range of the relevant phase space.
}

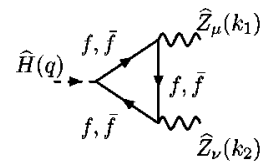

(a)

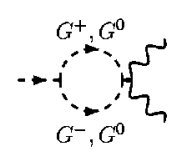

(b3)

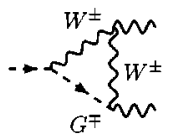

(c2)

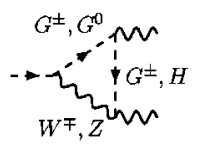

(c5)

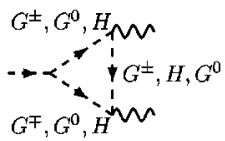

(c8)

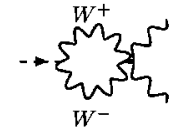

(b1)

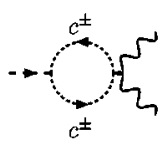

(b4)

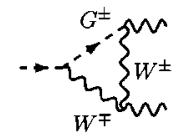

(c3)

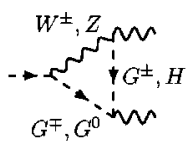

(c6)

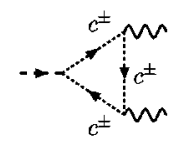

(c9)

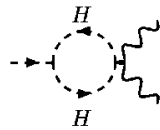

(b2)

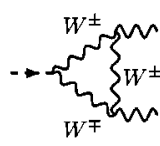

(c1)

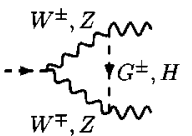

(c4)

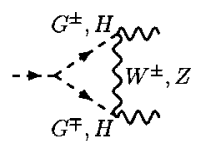

(c7)

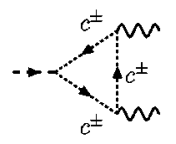

(c10)
FIG. 2. Diagrams contributing to the one-loop vertex $\hat{\Gamma}_{\mu \nu}^{H Z Z}$.

$$
\begin{aligned}
& \hat{M}_{s s}=s|\Delta|^{2} m_{f}^{2} \beta_{f}^{2} \hat{f}_{1}, \\
& \hat{M}_{s t}=\frac{2 s^{2}}{\left(t-m_{f}^{2}\right)}|\Delta|^{2} m_{f}^{2}\left[\hat{f}_{2} \beta_{f}^{2}-\hat{f}_{3} z x\right], \\
& \hat{M}_{s u}=\frac{2 s^{2}}{\left(u-m_{f}^{2}\right)}|\Delta|^{2} m_{f}^{2}\left[\hat{f}_{2} \beta_{f}^{2}+\hat{f}_{3} z x\right],
\end{aligned}
$$

where

$$
|\Delta|^{2}=\left[\left(s-M_{H}^{2}\right)^{2}+s^{2} L^{2}\right]^{-1},
$$

and

$$
\begin{aligned}
\hat{f}_{1}= & f_{1}+\frac{s^{2}}{M_{Z}^{4}}(L-R)^{2}-4 \frac{s}{M_{Z}^{2}}[R(A+B)+A L] \\
& +4\left(3 A^{2}+B^{2}+2 A B\right), \\
\hat{f}_{2}= & f_{2}\left(1-\frac{M_{H}^{2}}{s}\right)+B L+\left(3-4 a_{f}\right) A L-\frac{s}{M_{Z}^{2}} L R \\
\hat{f}_{3}= & f_{3}\left(1-\frac{M_{H}^{2}}{s}\right)+\left(1+4 a_{f}\right) L R+2 \frac{m_{f}^{2}}{M_{Z}^{2}} A L \\
& -\frac{s}{M_{Z}^{2}} L\left(R-\frac{m_{f}^{2}}{M_{Z}^{2}}(R-L)\right),
\end{aligned}
$$


with

$$
R \equiv A+\frac{1}{2} B
$$

Similarly, the total cross section is given from Eq. (2.14) after replacing $\sigma_{s s}, \sigma_{s t}$ and $\sigma_{s u}$ by $\hat{\sigma}_{s s}, \hat{\sigma}_{s t}$, and $\hat{\sigma}_{s u}$, respectively, given by

$$
\begin{gathered}
\hat{\sigma}_{s s}=2 s|\Delta|^{2} m_{f}^{2} \beta_{f}^{2} \hat{f}_{1}, \\
\hat{\sigma}_{s t}=\hat{\sigma}_{s u}=-8 s|\Delta|^{2} m_{f}^{2}\left[\hat{f}_{3}-\frac{V}{2 z}\left(\beta_{f}^{2} \hat{f}_{2}+y \hat{f}_{3}\right)\right] .
\end{gathered}
$$

Clearly, only the purely $s$-channel contributions together with the interference terms are modified, while the "genuine" $t$ - and $u$ - channel contributions (box-like terms) remain unaffected.

It is now obvious from Eq. (3.6) that one cannot recover the correct high energy behavior of the amplitude for generic values of the functions $L, A$, and $B$. For example, even if we choose the $L, A$, and $B$ such that asymptotically $L=R$, if the individual $L, A$, and $B$ grow sufficiently fast with $s$ the resulting amplitude has the wrong large- $s$ limit. Reversing the situation, in general even if the individual $L, A$, and $B$ are assumed not to grow faster than constants, unless we also have that $L \rightarrow R$, the resulting total cross section will behave asymptotically at least as a constant, instead of the correct behavior given in Eq. (2.17). ${ }^{5}$

Let us now turn to the $L, A$ and $B$ derived within the PT. ${ }^{6}$ Using the PT [5] one can reorganize the one-loop $S$-matrix in such a way as to define Higgs boson and Goldstone boson self-energies, $\hat{\Pi}\left(q^{2}\right)$ and $\hat{\Pi}^{G^{0} G^{0}}\left(q^{2}\right)$, respectively, and $H Z Z$ and $H G^{0} G^{0}$ vertices, $\hat{\Gamma}_{\mu \nu}^{H Z Z}$ and $\hat{\Gamma}^{H G^{0} G^{0}}$, respectively, endowed with all the important properties listed in the Introduction. In particular, (i) asymptotically $\hat{L}$ goes to a constant, whereas $\hat{A}$ and $\hat{B}$ grow logarithmically (this has been established in [5] and is also studied in detail in the next section), (ii) they are related by the following tree-level $\mathrm{WI}^{7}$

$$
\begin{aligned}
k_{1}^{\mu} k_{2}^{\nu} \hat{\Gamma}_{\mu \nu}^{H Z Z}+M_{Z}^{2} \hat{\Gamma}^{H G^{0} G^{0}}= & \frac{i g_{w} M_{Z}}{2 c_{w}} \\
& \times\left[\hat{\Pi}\left(q^{2}\right)+\hat{\Pi}^{G^{0} G^{0}}\left(k_{1}^{2}\right)\right. \\
& \left.+\hat{\Pi}^{G^{0} G^{0}}\left(k_{2}^{2}\right)\right] .
\end{aligned}
$$

\footnotetext{
${ }^{5} \mathrm{~A}$ physically relevant counter-example is the case where $A=B$ $=0$ and $L=\mathrm{const} / \mathrm{s}$, which will be studied at the end of this section.

${ }^{6}$ In what follows we will use "hats" to indicate all such quantities.

${ }^{7}$ To see that Eq. (3.9) has indeed the same form as its tree-level counterpart notice that for $\hat{\Gamma}_{\mu \nu}^{H Z Z} \rightarrow \hat{\Gamma}_{0 \mu \nu}^{H Z Z}, \hat{\Pi}\left(q^{2}\right) \rightarrow\left(q^{2}-M_{H}^{2}\right)$, $\hat{\Gamma}^{H G^{0} G^{0}} \rightarrow \Gamma_{0}^{H G^{0} G^{0}}=i g_{w} M_{Z} M_{H}^{2} / 2 c_{w}$, and $\hat{\Pi}^{G^{0} G^{0}}\left(M_{Z}^{2}\right) \rightarrow M_{Z}^{2}$ one recovers the WI of Eq. (2.2).
}

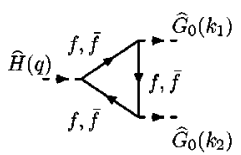

(a)

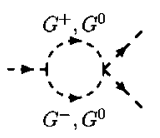

(d)

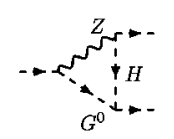

(g)

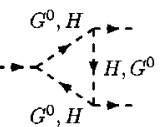

(j)

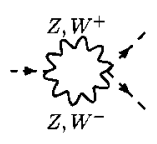

(b)

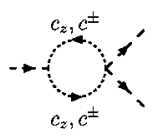

(e)

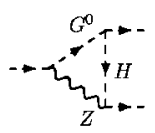

(b)

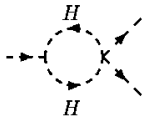

(c)

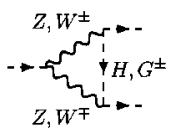

(f)

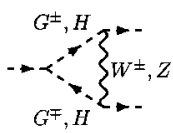

(i)
FIG. 3. Diagrams contributing to the one-loop vertex $\hat{\Gamma}^{H G^{0} G^{0}}$.

We are now in position to study explicitly how the correct high energy behavior of the Born-improved amplitude is enforced. First of all, by virtue of the first property listed above, i.e. because the individual $\hat{L}, \hat{A}$, and $\hat{B}$ grow mildly with $s$, we only need to show that $L=R$ asymptotically. To see how this comes about, we start with the parametrization of $\hat{\Gamma}_{\mu \nu}^{H Z Z}$ given in Eq. (3.3) and act with $k_{1}^{\mu} k_{2}^{\nu}$ on both sides; in the limit $s \gg M_{Z}^{2}$, we obtain

$$
k_{1}^{\mu} k_{2}^{\nu} \hat{\Gamma}_{\mu \nu}^{H Z Z}=\frac{i g_{w} M_{Z}}{2 c_{w}} s \hat{R} .
$$

On the other hand, putting the Z's on shell in Eq. (3.9) and using the form of $\hat{\Pi}^{H H}(s)$ given in Eq. (3.1), we have that

$$
k_{1}^{\mu} k_{2}^{\nu} \hat{\Gamma}_{\mu \nu}^{H Z Z}=\frac{i g_{w} M_{Z}}{2 c_{w}}\left[\hat{L} s+2 \hat{\Pi}^{G^{0} G^{0}}\left(M_{Z}^{2}\right)\right]-M_{Z}^{2} \hat{\Gamma}^{H G^{0} G^{0}} .
$$

Setting

$$
\hat{\Gamma}^{H G^{0} G^{0}}=\frac{i g_{w} M_{Z}}{2 c_{w}} \hat{D}(s),
$$

we obtain after equating the left-hand sides of Eq. (3.10) and Eq. (3.11)

$$
\hat{R}-\hat{L}=\frac{1}{s}\left[2 \hat{\Pi}^{G^{0} G^{0}}\left(M_{Z}^{2}\right)-M_{Z}^{2} \hat{D}(s)\right] .
$$

Thus, the role of the WI is to supply this last relation; however, no additional information about the high energy behavior of either side of Eq. (3.13) is provided.

The next important step is to establish that the terms inside the parenthesis on the left-hand side of Eq. (3.13) grow at most logarithmically for large values of $s$. Indeed, to begin with, $\hat{\Pi}^{G^{0} G^{0}}\left(M_{Z}^{2}\right)$ is a constant, independent of $s$. On the 
other hand, $\hat{D}(s)$ has a non-trivial dependence on $s$; using the diagrams of Fig. 3, together with the Feynman rules given in $[20]^{8}$ and Eq. (A6) in the Appendix, one can verify that

$$
\hat{D}(s) \sim \ln \left(s / \mu^{2}\right)+\cdots,
$$

and thus, from Eq. (3.13)

$$
\hat{R}-\hat{L}=0+\mathcal{O}\left(s^{-1} \ln \left(s / \mu^{2}\right)\right) .
$$

As mentioned before, this last relation is crucial for recovering the correct asymptotic behavior for the amplitude. Indeed, in the limit $s \gg \mu^{2}$, setting $L=R$ in Eq. (3.13) yields

$$
\begin{aligned}
& \hat{f}_{1}=-8 \frac{s}{M_{Z}^{2}}\left(1+\hat{R}^{2}\right)+\cdots, \\
& \hat{f}_{2}=-\frac{s}{M_{Z}^{2}}\left(1+\hat{R}^{2}\right)+\cdots, \\
& \hat{f}_{3}=-\frac{s}{M_{Z}^{2}}\left(1+\hat{R}^{2}\right)+\cdots
\end{aligned}
$$

Thus, as happens in the non-resonant case of the previous section $\hat{f}_{2}=\hat{f}_{3}=\frac{1}{8} \hat{f}_{1}$ and therefore

$$
\hat{\sigma}_{s s}+\hat{\sigma}_{s t}+\hat{\sigma}_{s u}=\mathcal{O}\left(s^{-1} \ln \left(s / \mu^{2}\right)\right) .
$$

We are now in a position to fully appreciate the role of the WI. Even though asymptotically $\hat{L}$ goes to a constant, whereas $\hat{A}$ and $\hat{B}$ grow logarithmically (a fact which, without additional information, would make us infer that the high energy behavior of the amplitude would be distorted) delicate cancellations make the crucial quantity $(L-R)$ energetically suppressed. Thus, cancellations taking place on the lefthand side of Eq. (3.13), whose study would necessitate explicit knowledge of $\hat{L}, \hat{A}$ and $\hat{B}$, are directly encoded in the $\hat{D}$ appearing on the right-hand side.

The conclusion of this analysis is that by virtue of the one-loop Ward identities and the good individual highenergy behavior of the PT one-loop Green's functions the tree-level and Born-improved amplitudes coincide sufficiently far away from the resonance region.

We end this section by studying the behavior of the Bornimproved amplitude for two different choices for the parameters $A, B$ and $L$; these choices are not just arbitrary mathematical examples, but have instead a rather well-known field-theoretical origin. In the first case the WI is violated but the correct high energy behavior of the amplitude is none-

\footnotetext{
${ }^{8}$ Here we are using the well-known fact [21] that at one-loop the PT effective Green's functions coincide with the conventional (gauge-fixing-parameter-dependent) Green's functions of the background field method, for the special value $\xi_{Q}=1$. This correspondence does not persist beyond one loop [22].
}

theless recovered; in the second case the WI is satisfied, but the high energy behavior is distorted. The first example corresponds to the case where a constant width is used in the Born-improved amplitude, i.e. the Higgs boson width has the form $\Im m \Pi=\Sigma_{i} c_{i} \theta\left(s-4 M_{i}^{2}\right)$, where the coefficients $c_{i}$ are constants, independent of $s$; their exact expressions may be obtained from Eq. (4.1) by setting $s=M_{H}^{2}$. In addition we make the simplest assumption for the vertex, namely $A=B$ $=0$; clearly, such an assumption violates the WI given in Eq. (2.2). For large values of $s$ we than have that $\Im m \Pi=\Sigma_{i} c_{i}$ $\equiv c$. In that case we have that $L=c / s$ and the corresponding expressions for the $\hat{f}_{1}, \hat{f}_{2}$, and $\hat{f}_{3}$ reduce to

$$
\begin{aligned}
& \hat{f}_{1}=f_{1}+\frac{c^{2}}{M_{Z}^{4}}, \quad \hat{f}_{2}=f_{2}\left(1-\frac{M_{H}^{2}}{s}\right), \\
& \hat{f}_{3}=f_{3}\left(1-\frac{M_{H}^{2}}{s}\right)+\frac{c^{2}}{M_{Z}^{4}} \frac{m_{f}^{2}}{s} .
\end{aligned}
$$

So, in the limit of large $s$ we recover the correct asymptotic relation for the $\hat{f}_{i}$, and therefore for the entire cross section. Of course, the use of a constant width is known to be problematic for other reasons, for example the fact that it leads to a violation of the optical theorem [23] (for details see the second paper of [4]).

The second case pertains to the unitary gauge (the $\xi \rightarrow \infty$ limit of the renormalizable $R_{\xi}$ gauges). In this gauge the WI of Eq. (3.9) is satisfied by the conventional two and three point functions, before resorting to the PT algorithm [24]; however, their imaginary parts display a strong dependence on $s$. For example the running width corresponding to two virtual $W$ bosons in the unitary gauge is given by $\Im m \Pi^{(\infty)}$ $\sim\left(g^{2} / M_{W}^{2}\right)\left(s^{2}-4 s M_{W}^{2}+12 M_{W}^{4}\right)$, and so, for large $s$ we have that $L \sim\left(g^{2} / M_{W}^{2}\right) s$, which leads to a gross distortion of the high energy behavior of the Born-improved amplitude.

\section{EXPLICIT REALIZATION IN THE PINCH TECHNIQUE FRAMEWORK}

In this section we will show explicitly that the PT selfenergies and vertices satisfy the required relations at high energies. In particular we will prove the validity of Eq. (3.13) without resorting to the WI of Eq. (3.9) as we did in the previous section, but instead by showing directly that, for asymptotic values of $s, \hat{L}=\hat{R}$. This calculation constitutes a non-trivial test of the entire construction; for practical purposes it is essential, given the fact that the Green's functions related to the unphysical Goldstone bosons, while they are crucial for realising the WI of Eq. (3.9), do not explicitly appear in the actual computation of the cross section. Indeed, the only quantities which appear in Eq. (3.6) are the $\hat{A}, \hat{B}$, and $\hat{L}$, but not the $\hat{D}$ of Eq. (3.12) nor the $\hat{\Pi}^{G^{0} G^{0}}$.

We next proceed with the explicit calculation. The partial running widths for the Higgs boson have been first calculated at one-loop in [5]; they are given by 


$$
\begin{aligned}
\Im m \hat{\Pi}_{(W W)}(s)= & \frac{\alpha_{w}}{16} \frac{M_{H}^{4}}{M_{W}^{2}}\left[1+4 \frac{M_{W}^{2}}{M_{H}^{2}}-4 \frac{M_{W}^{2}}{M_{H}^{4}}\left(2 s-3 M_{W}^{2}\right)\right] \\
& \times \beta_{W} \theta\left(s-4 M_{W}^{2}\right), \\
\Im m \hat{\Pi}_{(Z Z)}(s)= & \frac{\alpha_{w}}{32} \frac{M_{H}^{4}}{M_{W}^{2}}\left[1+4 \frac{M_{Z}^{2}}{M_{H}^{2}}-4 \frac{M_{Z}^{2}}{M_{H}^{4}}\left(2 s-3 M_{Z}^{2}\right)\right] \\
& \times \beta_{Z} \theta\left(s-4 M_{Z}^{2}\right), \\
\Im m \hat{\Pi}_{(F F)}(s)= & N_{F} \frac{\alpha_{w}}{8} \frac{m_{F}^{2}}{M_{W}^{2}} s \beta_{F}^{3} \theta\left(s-4 m_{F}^{2}\right), \\
\Im m \hat{\Pi}_{(H H)}(s)= & \frac{9 \alpha_{w}}{32} \frac{M_{H}^{4}}{M_{W}^{2}} \beta_{H} \theta\left(s-4 M_{H}^{2}\right) .
\end{aligned}
$$

In the above formula we denote by $F$ the various fermionic flavors appearing inside the quantum loops, i.e. $F$ $\in\{e, \mu, \tau, u, d, c, s, t, b\} . N_{F}=1$ for leptons, and $N_{F}=3$ for quarks. In the case of a heavy Higgs boson the channels which dominate numerically are the $W W, Z Z$ and $t t$. From the above expressions we can extract the dimensionless quantities $\hat{L}^{(W W)}, \hat{L}^{(Z Z)}, \hat{L}^{(F F)}$, and $\hat{L}^{(H H)}$, according to the definition of Eq. (3.2); in the limit of large $s$ they will be simply the coefficients multiplying $s$ in the expressions given on the right-hand side of Eq. (4.1).

The absorptive form factors $\hat{A}$ and $\hat{B}$ of the $H Z Z$ one-loop vertex are obtained from the graphs shown in Fig. 2, using the Feynman rules of [20], and can be expressed in terms of the standard Passarino-Veltman one-loop integrals [25] as given in [26]. For on shell external $Z$ bosons the arguments of the $C$ functions appearing in the calculation are $C\left(M_{Z}^{2}, M_{Z}^{2}, s, m_{i}^{2}, m_{j}^{2}, m_{i}^{2}\right)$, where $m_{i}, m_{j}$ are the masses of the particles inside the triangle, $(i j i) \in\{(F F F),(W W W),(Z H Z),(H Z H)\}$. We will use the shorthand notation where the first three (common) arguments will be suppressed, and the remaining three masses will be denoted as a superscript, i.e. $C\left(M_{Z}^{2}, M_{Z}^{2}, s, m_{i}^{2}, m_{j}^{2}, m_{i}^{2}\right)$ $\equiv C^{(i j i)}$. Similarly, for the $B_{0}$ functions we use the shorthand notation $B_{0}\left(s, m_{i}, m_{i}\right) \equiv B_{0}^{(i i)}$. Finally, a "bar" over $B_{0}$ and $C$ indicates that only their absorptive part has been considered. The individual diagrams yield ${ }^{9}$

$$
\begin{aligned}
i \hat{A}_{(a)}= & N_{F} \frac{\alpha_{w}}{8 \pi} \frac{m_{t}^{2}}{M_{W}^{2}}\left\{a _ { F } \left[s \beta_{Z}^{2}\left(\bar{C}_{0}^{(F F F)}+2 \bar{C}_{11}^{(F F F)}\right)\right.\right. \\
& \left.-8 \bar{C}_{24}^{(F F F)}\right]+\frac{1}{4}\left[s\left(\beta_{Z}^{2}+2 \beta_{F}^{2}\right) \bar{C}_{0}^{(F F F)}\right. \\
& \left.\left.+2 s \beta_{Z}^{2} \bar{C}_{11}^{(F F F)}-8 \bar{C}_{24}^{(F F F)}\right]\right\}
\end{aligned}
$$

${ }^{9}$ The closed expression for the PT absorptive form factors $\hat{A}$ and $\hat{B}$ have been presented first in [5], but here we correct several misprints.

$$
\begin{aligned}
i \hat{A}_{(b 1)}= & -\frac{2 \alpha_{w}}{\pi} \frac{M_{W}^{4}}{M_{Z}^{4}} \bar{B}_{0}^{(W W)}, \\
i \hat{A}_{(b 2)}= & -\frac{\alpha_{w}}{16 \pi} \frac{3}{2} \frac{M_{H}^{2}}{M_{W}^{2}} \bar{B}_{0}^{(H H)}, \\
i \hat{A}_{(b 3)}= & -\frac{\alpha_{w}}{16 \pi}\left[\left(\frac{M_{H}^{2}}{M_{W}^{2}}+2\right)\left(2 \frac{M_{W}^{2}}{M_{Z}^{2}}-1\right)^{2} \bar{B}_{0}^{(W W)}\right. \\
& \left.+\frac{1}{2}\left(\frac{M_{H}^{2}}{M_{W}^{2}}+2 \frac{M_{Z}^{2}}{M_{W}^{2}}\right) \bar{B}_{0}^{(Z Z)}\right], \\
i \hat{A}_{(b 4)}= & \frac{\alpha_{w}}{\pi} \frac{M_{W}^{4}}{M_{Z}^{4}} \bar{B}_{0}^{(W W)}, \\
i \hat{A}_{(c 1)}= & \frac{2 \alpha_{w}}{\pi} \frac{M_{W}^{4}}{M_{Z}^{4}}\left[4 \bar{C}_{24}^{(W W W)}+\left(s-2 M_{Z}^{2}\right) \bar{C}_{0}^{(W W W)}\right],
\end{aligned}
$$$$
i \hat{A}_{(c 2)}+i \hat{A}_{(c 3)}=-\frac{\alpha_{w}}{\pi} \frac{M_{W}^{2}}{M_{Z}^{2}} s \bar{C}_{0}^{(W W W)},
$$$$
i \hat{A}_{(c 4)}=\frac{\alpha_{w}}{4 \pi}\left[2 M_{W}^{2} \bar{C}_{0}^{(W W W)}+\frac{M_{Z}^{4}}{M_{W}^{2}} \bar{C}_{0}^{(Z H Z)}\right],
$$$$
i \hat{A}_{(c 5)}=i \hat{A}_{(c 6)}=0 \text {, }
$$$$
i \hat{A}_{(c 7)}=\frac{\alpha_{w}}{8 \pi}\left[2\left(M_{H}^{2}+2 M_{W}^{2}\right) \bar{C}_{0}^{(W W W)}\right.
$$$$
\left.+3 M_{Z}^{2} \frac{M_{H}^{2}}{M_{W}^{2}} \bar{C}_{0}^{(H Z H)}\right] \text {, }
$$$$
i \hat{A}_{(c 8)}=\frac{\alpha_{w}}{4 \pi}\left[\left(2 \frac{M_{W}^{2}}{M_{Z}^{2}}-1\right)^{2}\left(\frac{M_{H}^{2}}{M_{W}^{2}}+2\right) \bar{C}_{24}^{(W W W)}\right.
$$$$
+\frac{1}{2}\left(\frac{M_{H}^{2}}{M_{W}^{2}}+2 \frac{M_{Z}^{2}}{M_{W}^{2}}\right) \bar{C}_{24}^{(Z H Z)}
$$$$
\left.+\frac{3}{2} \frac{M_{H}^{2}}{M_{W}^{2}} \bar{C}_{24}^{(H Z H)}\right] \text {, }
$$$$
i \hat{A}_{(c 9)}+i \hat{A}_{(c 10)}=-\frac{4 \alpha_{w}}{\pi} \frac{M_{W}^{4}}{M_{Z}^{4}} \bar{C}_{24}^{(W W W)} .
$$

The individual contributions to the $B$ form factor are given by 


$$
\begin{aligned}
& i \hat{B}_{(a)}=-N_{F} \frac{\alpha_{w}}{4 \pi} \frac{m_{F}^{2}}{M_{W}^{2}} s\left\{a _ { F } \left[3 \bar{C}_{0}^{(F F F)}+4 \bar{C}_{11}^{(F F F)}\right.\right. \\
& \left.\left.-4 \bar{C}_{23}^{(F F F)}\right]+\frac{1}{4}\left[\bar{C}_{0}^{(F F F)}-4 \bar{C}_{23}^{(F F F)}\right]\right\}, \\
& i \hat{B}_{(b 1)}=i \hat{B}_{(b 2)}=i \hat{B}_{(b 3)}=i \hat{B}_{(b 4)}=i \hat{B}_{(c 4)}=i \hat{B}_{(c 7)} \\
& =0 \text {, } \\
& i \hat{B}_{(c 1)}=-\frac{4 \alpha_{w}}{\pi} s \frac{M_{W}^{4}}{M_{Z}^{4}}\left[2 \bar{C}_{12}^{(W W W)}+2 \bar{C}_{23}^{(W W W)}\right. \\
& \left.+\bar{C}_{0}^{(W W W)}\right] \\
& i \hat{B}_{(c 2)}+i \hat{B}_{(c 3)}=-\frac{2 \alpha_{w}}{\pi} s \frac{M_{W}^{2}}{M_{Z}^{2}} \bar{C}_{11}^{(W W W)}, \\
& i \hat{B}_{(c 5)}+i \hat{B}_{(c 6)}=-\frac{\alpha_{w}}{2 \pi} \frac{s}{M_{Z}^{2}}\left[2\left(2 M_{W}^{2}-M_{Z}^{2}\right) \bar{C}_{12}^{(W W W)}\right. \\
& \left.-\frac{M_{Z}^{4}}{M_{W}^{2}} \bar{C}_{12}^{(Z H Z)}\right] \\
& i \hat{B}_{(c 8)}=-\frac{\alpha_{w}}{4 \pi} s\left[\left(2 \frac{M_{W}^{2}}{M_{Z}^{2}}-1\right)^{2}\left(\frac{M_{H}^{2}}{M_{W}^{2}}+2\right)\right. \\
& \times\left(\bar{C}_{12}^{(W W W)}+\bar{C}_{23}^{(W W W)}\right) \\
& +\frac{1}{2}\left(\frac{M_{H}^{2}}{M_{W}^{2}}+2 \frac{M_{Z}^{2}}{M_{W}^{2}}\right)\left(\bar{C}_{12}^{(Z H Z)}+\bar{C}_{23}^{(Z H Z)}\right) \\
& \left.+\frac{3}{2} \frac{M_{H}^{2}}{M_{W}^{2}}\left(\bar{C}_{12}^{(H Z H)}+\bar{C}_{23}^{(H Z H)}\right)\right] \\
& i \hat{B}_{(c 9)}+i \hat{B}_{(c 10)}=\frac{4 \alpha_{w}}{\pi} s \frac{M_{W}^{4}}{M_{Z}^{4}}\left[\bar{C}_{12}^{(W W W)}+\bar{C}_{23}^{(W W W)}\right] .
\end{aligned}
$$

In deriving the above results we have also used the identity

$$
\bar{C}_{0}^{(i j i)}+\bar{C}_{11}^{(i j i)}+\bar{C}_{12}^{(i j i)}=0 .
$$

From the Eq. (4.2) and Eq. (4.3) we may collect the total contribution of each separate channel to $\hat{A}$ and $\hat{B}$, which will be denoted by $\hat{A}^{(i j i)}$ and $\hat{B}^{(i j i)}$; using the definition in Eq. (3.7) we may then construct the corresponding $\hat{R}^{(i j i)}$. In order to determine their asymptotic behavior, we must use that in the limit of large $s$

$$
\bar{C}_{11}^{(i j i)} \rightarrow-\frac{1}{s} \bar{B}_{0}^{(i i)}, \quad \bar{C}_{23}^{(i j i)} \rightarrow \frac{1}{s} \bar{B}_{0}^{(i i)}, \quad \bar{C}_{24}^{(i j i)} \rightarrow \frac{1}{4} \bar{B}_{0}^{(i i)},
$$

as one may easily verify using the formulas presented in the Appendix. Thus we arrive at the following limits for the various $\hat{R}$ :

$$
\begin{gathered}
\hat{R}^{(F F F)} \rightarrow N_{F} \frac{\alpha_{w}}{8 \pi} \frac{m_{F}^{2}}{M_{W}^{2}} \bar{B}_{0}^{(F F)} \rightarrow \frac{\alpha_{w}}{8} \frac{m_{F}^{2}}{M_{W}^{2}}=\hat{L}^{(F F)}, \\
\hat{R}^{(W W W)} \rightarrow-\frac{\alpha_{w}}{2 \pi} \bar{B}_{0}^{(W W)} \rightarrow-\frac{\alpha_{w}}{2}=\hat{L}^{(W W)}, \\
\hat{R}^{(Z H Z) \rightarrow-} \frac{\alpha_{w}}{4 \pi} \frac{M_{Z}^{2}}{M_{W}^{2}} \bar{B}_{0}^{(Z Z)} \rightarrow-\frac{\alpha_{w}}{4} \frac{M_{Z}^{2}}{M_{W}^{2}}=\hat{L}^{(Z Z)}, \\
\hat{R}^{(H Z H)} \rightarrow 0=\hat{L}^{(H H)} .
\end{gathered}
$$

This is the announced result. We notice that all necessary cancellations which lead to the desired result take place channel by channel, as one would expect on physical grounds. It is also important to emphasize that the explicit expressions for the $\hat{L}, \hat{A}$ and $\hat{B}$ derived in this section allow for a detailed study of the amplitude for the entire range of $s$, and not only asymptotically, as we have done here.

\section{CONCLUSIONS}

In this paper we have we shown that within the PT resummation formalism the resonant and asymptotic regions of processes with gauge bosons in the final state can be described correctly and connected to each other smoothly by means of a single Born-improved amplitude. In particular, using the resonant process $f \bar{f} \rightarrow Z Z$ as a reference process, we have studied in detail the mechanism which enforces the correct high-energy behavior of the Born-improved amplitude, and we have shown how this mechanism is in fact automatically (but non-trivially) realized in the PT framework. This provides an additional self-consistency check for the resummation formalism based on the PT.

An important by-product of this analysis is that explicit closed expression for the differential and total cross sections of the process $f \bar{f} \rightarrow Z Z$ have been computed, both at the tree level and in the Born-improved approximation. For the latter case the generic form of the amplitude for arbitrary selfenergy and vertex corrections has been reported, as well as the specific corrections obtained from the PT effective Green's function. Of course, if $M_{H}>2 M_{Z}$ the channel $f \bar{f}$ $\rightarrow W^{+} W^{-}$will be also relevant; however, the analysis presented in this paper may be carried out straightforwardly to the latter process, with the additional technical complications of computing the $Z$ and $\gamma$ mediated background.

In the present work we have only treated the case where the (two) gauge bosons appeared in the final state. The above considerations may be generalized to the case where both incoming and outgoing particles are gauge bosons. The $W$-fusion sub-process $W W \rightarrow Z Z$ for example has been recently studied for the case of off-shell W's [27]; it was shown how the tree-level PT rearrangement of the process $q q \rightarrow q q Z Z$ restores the good high energy behavior of the 


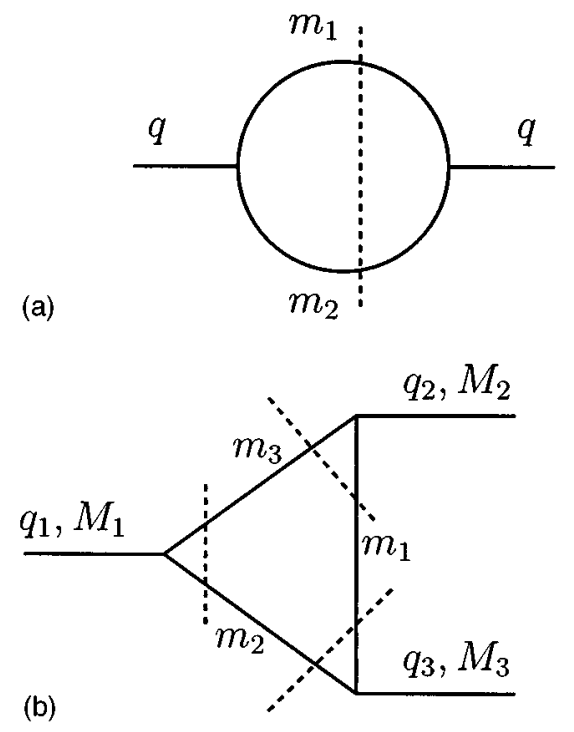

FIG. 4. The absorptive parts of the three-point function.

aforementioned sub-process, thus solving a long-standing problem [28]. Based on the analysis presented here one expects that the results established in [27] will persists after the one-loop PT corrections necessary for regulating the $W$-fusion amplitude near the Higgs boson resonance have been included.

Given the explicit results for the process $f \bar{f} \rightarrow Z Z$ presented in this paper one could carry out a detailed study of the standard model Higgs boson line shape, obtained from the above process. Such a study could be of potential interest in the context of a muon-collider, for example, but is beyond the scope of the present paper.

\section{ACKNOWLEDGMENTS}

This work has been funded by Grant No. TMRERBFMBICT 972024. I thank R. Pittau for independently checking several of the calculations appearing in this paper, and A. Pilaftsis and K. Philippides for various useful discussions.

\section{APPENDIX: ABSORPTIVE PARTS OF THE $B_{0}$ AND $C$ FUNCTIONS}

In this appendix we list some formulas which are useful when computing the absorptive (imaginary) parts of the $B_{0}$ and $C$ functions. 4a)

For the imaginary part of the $B_{0}$ function we have (Fig.

$$
\bar{B}_{0}\left(q^{2}, m_{1}^{2}, m_{2}^{2}\right)=\frac{1}{q^{2}} \pi \theta\left[q^{2}-\left(m_{1}+m_{2}\right)^{2}\right] \lambda^{1 / 2}\left(q^{2}, m_{1}^{2}, m_{2}^{2}\right),
$$

where $\lambda(x, y, z)=(x-y-z)^{2}-4 y z$. In the cases studied in this paper we have always $m_{1}=m_{2}=m$, and the above formula reduces to

$$
\bar{B}_{0}\left(q^{2}, m^{2}, m^{2}\right)=\pi \theta\left(q^{2}-4 m^{2}\right) \beta .
$$

The imaginary parts of $C_{0}$ in the general case are given by (Fig. 4b)

$$
\begin{aligned}
\bar{C}_{0}= & \frac{\pi}{2} \theta\left(q_{1}^{2}-\left(m_{2}+m_{3}\right)^{2}\right) \lambda^{-1 / 2}\left(q_{1}^{2}, M_{2}^{2}, M_{3}^{2}\right) \\
& \times \ln \left[\frac{\rho_{1}^{-}-m_{1}^{2}}{\rho_{1}^{+}-m_{1}^{2}}\right]+\text { c.p. },
\end{aligned}
$$

with

$$
\begin{aligned}
\rho_{1}^{ \pm}= & M_{2}^{2}+m_{3}^{2}-\frac{1}{2 q_{1}^{2}}\left[\left(q_{1}^{2}+M_{2}^{2}-M_{3}^{2}\right)\left(q_{1}^{2}+m_{3}^{2}-m_{2}^{2}\right)\right. \\
& \left. \pm \lambda^{1 / 2}\left(q_{1}^{2}, M_{2}^{2}, M_{3}^{2}\right) \lambda^{1 / 2}\left(q_{1}^{2}, m_{2}^{2}, m_{3}^{2}\right)\right]
\end{aligned}
$$

and the abbreviation c.p. means cyclic permutation with respect to $1,2,3$.

For the particular channels appearing in our calculations we have

$$
\begin{aligned}
& \bar{C}_{0}^{(W W W)}=\frac{\pi}{2} \frac{1}{s \beta_{Z}} \theta\left(s-4 M_{W}^{2}\right) \ln \left(\frac{1+\beta_{Z}^{2}-2 \beta_{Z} \beta_{W}}{1+\beta_{Z}^{2}+2 \beta_{Z} \beta_{W}}\right), \\
& \bar{C}_{0}^{(F F F)}=\frac{\pi}{2} \frac{1}{s \beta_{Z}} \theta\left(s-4 m_{F}^{2}\right) \ln \left(\frac{1+\beta_{Z}^{2}-2 \beta_{Z} \beta_{F}}{1+\beta_{Z}^{2}+2 \beta_{Z} \beta_{F}}\right), \\
& \bar{C}_{0}^{(H Z H)}=\frac{\pi}{2} \frac{1}{s \beta_{Z}} \theta\left(s-4 M_{H}^{2}\right) \ln \left(\frac{1+\beta_{H}^{2}-2 \beta_{Z} \beta_{H}}{1+\beta_{H}^{2}+2 \beta_{Z} \beta_{H}}\right), \\
& \bar{C}_{0}^{(Z H Z)}=\frac{\pi}{2} \frac{1}{s \beta_{Z}} \theta\left(s-4 M_{Z}^{2}\right) \ln \left(\frac{1-\beta_{H}^{2}}{1+4 \beta_{Z}^{2}-\beta_{H}^{2}}\right) .
\end{aligned}
$$

For large $s$ the above formulas reduce to

$$
\bar{C}_{0}^{(i j i)}=-\frac{\pi}{2} \theta\left(s-4 M_{i}^{2}\right) \frac{1}{s} \ln \left(s / M_{i}^{2}\right)
$$

Finally, using the formulas of [26], the exact expressions for the remaining $C$ functions are given by

$$
\begin{aligned}
\bar{C}_{12}^{(F F F)}= & \frac{1}{s \beta_{Z}^{2}}\left(M_{Z}^{2} \bar{C}_{0}^{(F F F)}-\bar{B}_{0}^{(F F)}\right), \\
\bar{C}_{23}^{(F F F)}= & \frac{1}{s^{3} \beta_{Z}^{4}}\left(\left[2 M_{Z}^{4}\left(M_{Z}^{2}-4 m_{F}^{2}\right)+2 s M_{Z}^{2}\left(M_{Z}^{2}+3 m_{F}^{2}\right)\right.\right. \\
& \left.-s^{2}\left(M_{Z}^{2}+m_{F}^{2}\right)\right] \bar{C}_{0}^{(F F F)} \\
& \left.+\left(2 M_{Z}^{4}-3 s M_{Z}^{2}+s^{2}\right) \bar{B}_{0}^{(F F)}\right),
\end{aligned}
$$




$$
\begin{aligned}
& \bar{C}_{24}^{(F F F)}=\frac{1}{s \beta_{Z}^{2}}\left(\left[2 m_{F}^{2} M_{Z}^{2}-\frac{1}{2} M_{Z}^{4}-\frac{1}{2} s m_{F}^{2}\right] \bar{C}_{0}^{(F F F)}\right. \\
& \left.+\left[\frac{1}{4} s-\frac{1}{2} M_{Z}^{2}\right] \bar{B}_{0}^{(F F)}\right) \\
& \bar{C}_{12}^{(W W W)}=\frac{1}{s \beta_{Z}^{2}}\left(M_{Z}^{2} \bar{C}_{0}^{(W W W)}-\bar{B}_{0}^{(W W)}\right), \\
& \bar{C}_{23}^{(W W W)}=\frac{1}{s^{3} \beta_{Z}^{4}}\left(\left[2 M_{Z}^{4}\left(M_{Z}^{2}-4 M_{W}^{2}\right)+2 s M_{Z}^{2}\left(M_{Z}^{2}\right.\right.\right. \\
& \left.\left.+3 M_{W}^{2}\right)-s^{2}\left(M_{Z}^{2}+M_{W}^{2}\right)\right] \bar{C}_{0}^{(W W W)} \\
& \left.+\left[2 M_{Z}^{4}-3 s M_{Z}^{2}+s^{2}\right] \bar{B}_{0}^{(W W)}\right), \\
& \bar{C}_{24}^{(W W W)}=\frac{1}{s \beta_{Z}^{2}}\left(\left[2 M_{Z}^{2} M_{W}^{2}-\frac{1}{2} M_{Z}^{4}-\frac{1}{2} s M_{W}^{2}\right] \bar{C}_{0}^{(W W W)}\right. \\
& \left.+\left[\frac{1}{4} s-\frac{1}{2} M_{Z}^{2}\right] \bar{B}_{0}^{(W W)}\right) \\
& \bar{C}_{12}^{(Z H Z)}=\frac{1}{s \beta_{Z}^{2}}\left(M_{H}^{2} C_{0}^{(Z H Z)}-\bar{B}_{0}^{(Z Z)}\right),
\end{aligned}
$$

$$
\begin{aligned}
& \bar{C}_{23}^{(Z H Z)}=\frac{1}{s^{3} \beta_{Z}^{4}}\left(\left[2 M_{Z}^{2} M_{H}^{2}\left(M_{H}^{2}-4 M_{Z}^{2}\right)+2 s M_{H}^{2}\left(5 M_{Z}^{2}\right.\right.\right. \\
& \left.\left.-M_{H}^{2}\right)-2 s^{2} M_{H}^{2}\right] \bar{C}_{0}^{(Z H Z)} \\
& \left.+\left(s-M_{Z}^{2}\right)\left(s-4 M_{Z}^{2}+2 M_{H}^{2}\right) \bar{B}_{0}^{(Z Z)}\right), \\
& \bar{C}_{24}^{(Z H Z)}=\frac{1}{s \beta_{Z}^{2}}\left(M_{H}^{2}\left[2 M_{Z}^{2}-\frac{1}{2} M_{H}^{2}-\frac{1}{2} s\right] \bar{C}_{0}^{(Z H Z)}\right. \\
& \left.+\left[\frac{1}{2} M_{H}^{2}-M_{Z}^{2}+\frac{1}{4} s\right] \bar{B}_{0}^{(Z Z)}\right), \\
& \bar{C}_{12}^{(H Z H)}=\frac{1}{s \beta_{Z}^{2}}\left(\left(2 M_{Z}^{2}-M_{H}^{2}\right) \bar{C}_{0}^{(H Z H)}-\bar{B}_{0}^{(H H)}\right), \\
& \bar{C}_{23}^{(H Z H)}=\frac{1}{s^{3} \beta_{Z}^{4}}\left(\left[2 M_{Z}^{2} M_{H}^{2}\left(M_{H}^{2}-4 M_{Z}^{2}\right)\right.\right. \\
& +2 s\left(2 M_{Z}^{2} M_{H}^{2}+3 M_{Z}^{4}-M_{H}^{4}\right) \\
& \left.+s^{2}\left(M_{H}^{2}-3 M_{Z}^{2}\right)\right] \bar{C}_{0}^{(H Z H)} \\
& \left.+\left(s-M_{Z}^{2}\right)\left(s-2 M_{H}^{2}\right) \bar{B}_{0}^{(H H)}\right), \\
& \bar{C}_{24}^{(H Z H)}=\frac{1}{s \beta_{Z}^{2}}\left(\left[2 M_{Z}^{2} M_{H}^{2}-\frac{1}{2} M_{H}^{4}-\frac{1}{2} s M_{Z}^{2}\right] \bar{C}_{0}^{(H Z H)}\right. \\
& \left.+\left[\frac{1}{4} s-\frac{1}{2} M_{H}^{2}\right] \bar{B}_{0}^{(H H)}\right) \text {. }
\end{aligned}
$$

[1] A. Sirlin, Phys. Rev. Lett. 67, 2127 (1991); Phys. Lett. B 267, 240 (1991); R.G. Stuart, ibid. 262, 113 (1991); 272, 353 (1991); Phys. Rev. Lett. 70, 3193 (1993); M. Nowakowski and A. Pilaftsis, Z. Phys. C 60, 121 (1993); A. Aeppli, G.J. van Oldenborgh, and D. Wyler, Nucl. Phys. B428, 126 (1994); H. Veltman, Z. Phys. C 62, 35 (1994); M. Beuthe, R. Gonzalez Felipe, G. Lopez Castro, and J. Pestieau, Nucl. Phys. B498, 55 (1997); L. Maiani and M. Testa, Ann. Phys. (N.Y.) 263, 353 (1998).

[2] D.A. Dicus and V.S. Mathur, Phys. Rev. D 7, 3111 (1973); W.W. Repko and C.J. Suchyta, Phys. Rev. Lett. 62, 859 (1989); S. Dawson and S. Willenbrock, Phys. Rev. D 40, 2880 (1989); A. Dobado, Phys. Lett. B 237, 457 (1990); G. Valencia and S. Willenbrock, Phys. Rev. D 46, 2247 (1992); M.H. Seymour, Phys. Lett. B 354, 409 (1995); A. Ghinculov and J.J. van der Bij, Nucl. Phys. B482, 59 (1996); D. Wackeroth and W. Hollik, Phys. Rev. D 55, 6788 (1997); M. Kuroda and D. Schildknecht, Nucl. Phys. B531, 24 (1998); M. Passera and A. Sirlin, Phys. Rev. D 58, 113010 (1998); B.A. Kniehl and A. Sirlin, Phys. Rev. Lett. 81, 1373 (1998).

[3] J.M. Cornwall, D.N. Levin, and G. Tiktopoulos, Phys. Rev. D 10, 1145 (1974); 11, 972 (1975); C.E. Vayonakis, Lett. Nuovo Cimento 17, 383 (1976); M.S. Chanowitz and M.K. Gaillard, Nucl. Phys. B261, 379 (1985); G.J. Gounaris, R. Kögerler, and
H. Neufeld, Phys. Rev. D 34, 3257 (1986).

[4] J. Papavassiliou and A. Pilaftsis, Phys. Rev. Lett. 75, 3060 (1995); Phys. Rev. D 53, 2128 (1996); 54, 5315 (1996).

[5] J. Papavassiliou and A. Pilaftsis, Phys. Rev. Lett. 80, 2785 (1998); Phys. Rev. D 58, 053002 (1998).

[6] J. M. Cornwall, in Proceedings of the French-American Seminar on Theoretical Aspects of Quantum Chromodynamics, Marseille, France, 1981, edited J.W. Dash (Centre de Physique Théorique, Marseille, 1982); Phys. Rev. D 26, 1453 (1982).

[7] J.M. Cornwall and J. Papavassiliou, Phys. Rev. D 40, 3474 (1989); J. Papavassiliou, ibid. 41, 3179 (1990); G. Degrassi and A. Sirlin, ibid. 46, 3104 (1992).

[8] J. Papavassiliou, E. de Rafael, and N.J. Watson, Nucl. Phys. B503, 79 (1997).

[9] N.J. Watson, Phys. Lett. B 349, 155 (1995).

[10] N.J. Watson, Nucl. Phys. B494, 388 (1997).

[11] K. Philippides and A. Sirlin, Nucl. Phys. B477, 59 (1996).

[12] V. Barger, M.S. Berger, J.F. Gunion, and T. Han, Phys. Rev. Lett. 78, 3991 (1997); 286, 1 (1997); J.F. Gunion, T. Han, and R. Sobey, Phys. Lett. B 429, 79 (1998).

[13] U. Baur and D. Zeppenfeld, Phys. Rev. Lett. 75, 1002 (1995).

[14] E.N. Argyres et al., Phys. Lett. B 358, 339 (1995).

[15] See, for example, L.F. Abbott, Nucl. Phys. B185, 189 (1981), and references therein. 
[16] Yu.L. Dokshitzer, D.I. Dyakonov, and S.I. Troyan, Phys. Rep. 58, 269 (1980); A. Andraši and J.C. Taylor, Nucl. Phys. B192, 283 (1981); D.M. Capper and G. Leibbrandt, Phys. Rev. D 25, 1002 (1982).

[17] R.W. Brown and K.O. Mikaelian, Phys. Rev. D 19, 922 (1979).

[18] K. Philippides and A. Sirlin, Phys. Lett. B 367, 377 (1996).

[19] A. Pilaftsis, Phys. Rev. Lett. 77, 4996 (1996); Nucl. Phys. B504, 61 (1997).

[20] A. Denner, S. Dittmaier, and G. Weiglein, Nucl. Phys. B440, 95 (1995).

[21] A. Denner, S. Dittmaier, and G. Weiglein, Phys. Lett. B 333, 420 (1994); S. Hashimoto, J. Kodaira, Y. Yasui, and K. Sasaki,
Phys. Rev. D 50, 7066 (1994); E. de Rafael and N. J. Watson (unpublished).

[22] N.J. Watson, hep-ph/9812202.

[23] See, for example, the second paper of [4].

[24] J. Papavassiliou and K. Philippides, Phys. Rev. D 52, 2355 (1995).

[25] G. 't Hooft and M. Veltman, Nucl. Phys. B153, 365 (1979); G. Passarino and M. Veltman, ibid. B160, 151 (1979).

[26] B.A. Kniehl, Phys. Rep. 240, 211 (1994).

[27] K. Philippides and W.J. Stirling, hep-ph/9901451.

[28] R. Kleiss and W.J. Stirling, Phys. Lett. B 182, 75 (1986); U. Baur and E.W.N. Glover, Nucl. Phys. B347, 12 (1990); Phys. Rev. D 44, 99 (1991). 